\title{
The Training Sequence Code Dependence of EDGE Receivers using Zero IF Sampling
}

\author{
Martin Krueger, Member, IEEE, Robert Denk, and Bin Yang, Member, IEEE
}

\begin{abstract}
In the mobile communication standard GSM/EDGE, the base station can select one of eight training sequence codes as the midamble of the downlink transmitted bursts. If the receiving mobile station uses zero intermediate frequency sampling, the channel estimation is sensitive to the DC offset and IQ gain/phase imbalance of the RF transceiver. This letter shows for a common class of channel estimators that the sensitivity depends on the selected training sequence code. This sensitivity can become significant for 8PSK modulation.
\end{abstract}

Index Terms-GSM/EDGE receiver, channel estimation, IQ gain/phase imbalance, DC offset, 8PSK modulation.

\section{INTRODUCTION}

$\mathbf{R}$ ECENTLY, the $2 \mathrm{G}$ standard GSM was enhanced by EDGE. In [1] a good overview on EDGE equalization concepts and suggested reading on the EDGE standard are given. The focus of this letter is the training sequence code (TSC) based channel estimation which is done before equalization. We restrict ourselves to zero IF sampling receivers, which are most common for EDGE mobile stations.

GSM originally used GMSK modulation and robust channel coding only. To achieve higher data rates, GPRS and later EDGE were introduced. In contrast to GPRS, EDGE additionally uses 8PSK modulation. Both apply coding schemes with various levels of redundancy which are chosen adaptively by the network. In case of channels with high SINR (up to $30 \mathrm{~dB}$ ), sensitive modulation and coding schemes with high data rates are chosen. Here RF impairments like IQ gain/phase imbalance and DC offset play an important role in addition to noise. In this letter we show how these impairments compromise the channel estimate which is an important step before demodulation of the received burst.

The importance of DC offset for EDGE equalizers has already been pointed out in [2] and [3]. The first joint channel and DC estimation algorithm was presented in [2]. A first analysis of the dependence of DC estimation error on training sequence code can be found in [4], whereas IQ imbalance is not adressed. The authors of [4] propose to use a small training sequence code dependent intermediate frequency (IF, approximately $10 \mathrm{kHz}$ ) instead of zero IF. As most EDGE receivers are not prepared for IF operation, we treat zero IF receivers in the present letter. We show that IQ imbalance can

M. Krueger is with Infineon Technologies AG, Munich, Germany (e-mail: martin.krueger@infineon.com).

R. Denk is with the Department of Mathematics and Statistics, University of Konstanz, Germany (e-mail: robert.denk@uni-konstanz.de).

B. Yang is with the Chair of System Theory and Signal Processing, University of Stuttgart, Germany (e-mail: bin.yang@LSS.uni-stuttgart.de). lead to a significant loss of receiver performance in addition to the loss caused by the DC offset.

In the following section we describe a model of the received EDGE burst that includes two major RF impairments: DC offset and IQ gain/phase imbalance. In Section III we analyze a linear joint channel and DC estimator. In contrast to [2], we derive separate explicit equations for the estimation of the DC and the channel impulse response. In Section IV we derive the error terms of this estimator from noise and IQ imbalance. In GSM/EDGE systems, the base station can select one of eight training sequence codes as midamble of the downlink transmitted bursts. We show that the selection of the training sequence has a significant influence on the channel estimation quality. The impact of these error terms on the bit error rate is shown by simulations. In Section V conclusions are drawn based on the results and analysis presented in the letter.

\section{Modelling of the Received EDGE Burst}

Deriving a complete transmission model for an EDGE burst is not within the scope of this letter. A good explanation can be found, e. g., in [1]. We focus on that part of the received signal that is used for a non-blind channel estimation in the mobile station for downlink reception.

Fig. 1(a) shows the principal blocks of the EDGE transmission system: transmitter, channel, radio frequency receiver, and baseband processor. To keep the model simple, the channel impulse response includes fading, pulse shaping, and all (digital and analog) filters in the transmit and receive path. The order of the FIR filter representing the channel impulse response is denoted by $L$. Concerning fading, we assume that the resulting channel impulse response is constant for the short period that is used for the channel estimation of one burst.

Fig. 1(b) shows a signal model for each block. All radio frequency signals are replaced by their baseband equivalent and all analog signals are replaced by their samples. We assume that the sampling rate is identical to the GSM/EDGE symbol rate $f_{T}=13 \mathrm{MHz} / 48$. Any interference outside the Nyquist interval $\left[-f_{T} / 2, f_{T} / 2\right]$ is assumed to be sufficiently suppressed by linear filters. Interference within the Nyquist interval is approximated by additive white Gaussian noise $\tilde{w}_{k}$. Moreover, we assume that the symbol-by-symbol rotation of $\phi=\pi / 2$ (GMSK approximated by rotated BPSK) or $\phi=$ $3 \pi / 8$ (8PSK) of the base station transmitter is compensated by de-rotation in the digital part of the receiver. Only those received samples that are used for channel estimation are considered. Those samples are a function of the $N=26$ training symbols $t_{k}$ that are summarized into a code word $\left[t_{0}, \ldots, t_{N-1}\right]$. Eight different training sequence codes are defined in the GSM/EDGE standard [5]. They are enumerated 
(a)

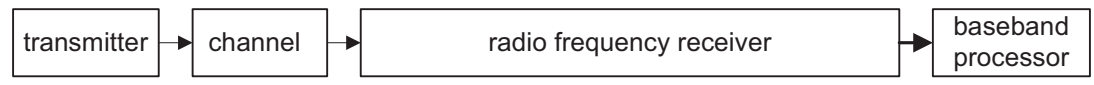

(b) rotation channel noise IQ gain and phase imbalance DC derotation

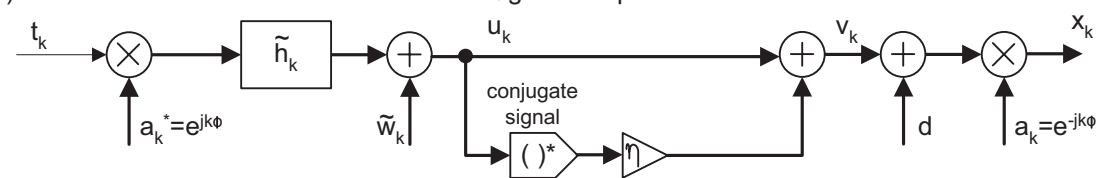

(c)

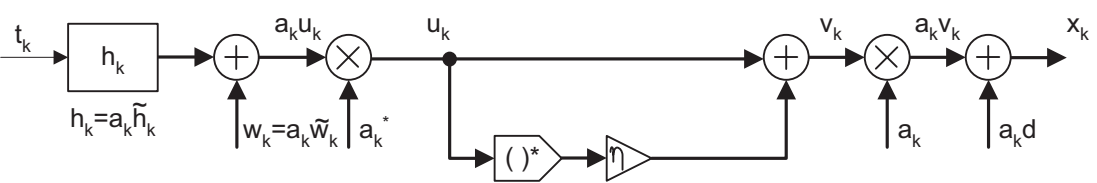

(d)
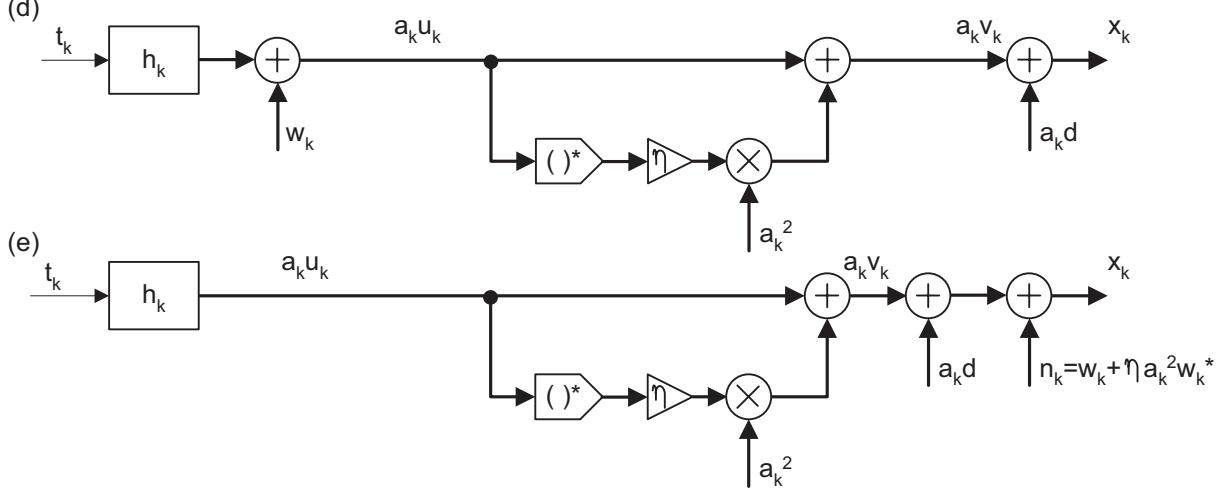

Fig. 1. Block diagram to derive the signal model.

from 0 to 7 and we use the same enumeration throughout this letter.

The discrete time is denoted by $k$. According to our model the input signal to the RF receiver $u_{k}$ is the convolution of the rotated training symbols with the overall channel impulse response $\tilde{h}_{k}$ plus additive white Gaussian noise $\tilde{w}_{k}$ with variance $N_{0}$. We obtain

$$
u_{k}=\sum_{\ell=0}^{L} \tilde{h}_{\ell} t_{k-\ell} e^{j(k-\ell) \phi}+\tilde{w}_{k}
$$

IQ gain/phase errors are added to $u_{k}$. When replacing the complex-valued samples with two-dimensional real-valued vectors, these can be modelled in a straightforward way [6], e. g. by applying different gains on I and Q component. To keep the benefits of the complex notation, we choose adding a mirror signal $\eta u_{k}^{*}$ instead, as suggested e. g. in [7] (p. 339, Eq. (4)), i. e. $v_{k}=u_{k}+\eta u_{k}^{*}$. Besides IQ imbalance the RF receiver adds a DC offset $d$. Eventually, the signal is de-rotated by $a_{k}=e^{-j k \phi}$, yielding demodulated output:

$$
x_{k}=a_{k}\left(v_{k}+d\right)=a_{k} v_{k}+a_{k} d \text {. }
$$

In Fig. 1, (c)+(d) show some rearrangements leading to an equivalent block diagram (e). The corresponding equation is

$$
x_{k}=\sum_{\ell=0}^{L} h_{\ell} t_{k-\ell}+\eta a_{k}^{2}\left(\sum_{\ell=0}^{L} h_{\ell} t_{k-\ell}\right)^{*}+a_{k} d+n_{k} .
$$

where

$$
n_{k}=w_{k}+\eta a_{k}^{2} w_{k}^{*}
$$

Since in $w_{k}$ real and imaginary part are uncorrelated it is straightforward to show that the variance of $n_{k}$ is $\left(1+|\eta|^{2}\right) N_{0}$. However, the real and imaginary part of $n_{k}$ are uncorrelated for $\eta=0$ only. In vector notation, Equations (3) and (4) are

$$
\mathbf{x}=\mathbf{T h}+\eta \mathbf{A}^{2} \mathbf{T h}^{*}+\mathbf{a} d+\mathbf{n},
$$

and

$$
\mathbf{n}=\mathbf{w}+\eta \mathbf{A}^{2} \mathbf{w}^{*}
$$

respectively. The real-valued $(N-L) \times(L+1)$-matrix is defined by

$$
\mathbf{T}=\left(\begin{array}{ccc}
t_{L} & \ldots & t_{0} \\
t_{L+1} & \ldots & t_{1} \\
\vdots & & \vdots \\
t_{N-1} & \ldots & t_{N-L-1}
\end{array}\right)
$$

The complex-valued column vectors $\mathbf{x}, \mathbf{h}, \mathbf{h}^{*}, \mathbf{a}$ and the complex-valued diagonal $(N-L) \times(N-L)$-matrix $\mathbf{A}$ are defined by

$$
\begin{aligned}
\mathbf{x} & =\left[\begin{array}{lll}
x_{L} & \ldots & x_{N-1}
\end{array}\right]^{T}, \\
\mathbf{h} & =\left[\begin{array}{lll}
h_{0} & \ldots & h_{L}
\end{array}\right]^{T}, \\
\mathbf{h}^{*} & =\left[\begin{array}{lll}
h_{0} & \ldots & h_{L}
\end{array}\right]^{H}, \\
\mathbf{a} & =\left[\begin{array}{lll}
e^{-j L \cdot \phi} & \ldots & e^{-j(N-1) \cdot \phi}
\end{array}\right]^{T} \\
\mathbf{A} & =\operatorname{diag}\{\mathbf{a}\}
\end{aligned}
$$

where $(\cdot)^{T}$ denotes transposition and $(\cdot)^{H}$ denotes conjugation plus transposition. 


\section{Channel Estimation Based on the TRAINING SEQUENCE CODE}

Based on Equation (5) a maximum likelihood channel estimator can be derived. A maximum likelihood channel estimator addresses DC offset and IQ imbalance and minimizes the error due to noise [6], [7]. Maximum likelihood joint estimation of the unknown imbalance parameter $\eta$ and the unknown channel impulse response $\mathbf{h}$ is a nonlinear problem and requires iterative processing. In frequency hopping channels, the RF impairments of the receiver can vary burst-byburst, so that the estimation has to be based on the $N-L$ samples of the current burst and has to be completed before equalization. Consequently, a maximum likelihood estimator seems not feasible for most GSM/EDGE mobile stations. To have an estimator that can be implemented with reasonable effort, we restrict ourselves to linear estimators.

In the following, we consider the maximum likelihood channel estimator for the case $\eta=0$. In this case $n_{k}$ is white Gaussian noise with variance $N_{0}$. Real and imaginary part of the noise have the same variance $N_{0} / 2$ and are uncorrelated. Hence, the joint maximum likelihood estimate for $\mathbf{h}$ and $d$ is given as the solution of the least squares problem

$$
\left(\begin{array}{l}
\hat{\mathbf{h}} \\
\hat{d}
\end{array}\right)=\arg \min _{\mathbf{h}, d}\left\|\left(\begin{array}{ll}
\mathbf{T} & \mathbf{a}
\end{array}\right)\left(\begin{array}{l}
\mathbf{h} \\
d
\end{array}\right)-\mathbf{x}\right\|^{2} .
$$

The solution is (see also [4])

$$
\left(\begin{array}{c}
\hat{\mathbf{h}} \\
\hat{d}
\end{array}\right)=\left(\mathbf{R}^{H} \mathbf{R}\right)^{-1} \mathbf{R}^{H} \mathbf{x}
$$

where $\mathbf{R}=\left(\begin{array}{ll}\mathbf{T} & \mathbf{a}\end{array}\right)$.

To understand the training sequence dependence of EDGE channel estimation, separate explicit expressions for the channel and DC estimation are useful. This separation can be obtained using the matrix inversion lemma. The result is

$$
\hat{d}=\mathbf{b}^{H} \mathbf{x}
$$

for the DC estimation where $\mathbf{b}=\mathbf{P}_{\mathbf{T}}^{\perp} \mathbf{a} /\left\|\mathbf{P}_{\mathbf{T}}^{\perp} \mathbf{a}\right\|^{2}, \mathbf{P}_{\mathbf{T}}^{\perp}=$ $\mathbf{I}-\mathbf{T} \mathbf{T}^{+}$, and $\mathbf{T}^{+}=\left(\mathbf{T}^{T} \mathbf{T}\right)^{-1} \mathbf{T}^{T}$. The channel impulse response is given by

$$
\hat{\mathbf{h}}=\mathbf{T}^{+}(\mathbf{x}-\mathbf{a} \hat{d}) .
$$

Please note that Equations (9) and (10) have the following properties:

1) They are mathematically equivalent to Equation (8).

2) They require fewer real-by-real multiplications than (8). Since $\mathbf{T}$ and $\mathbf{T}^{+}$are real-valued matrices, most complex-by-complex multiplications in (8) can be replaced by complex-by-real multiplications in (10).

3) They need less memory storage. Our method requires the storage of the real $(N-L) \times(L+1)$ matrix $\mathbf{T}^{+}$and the complex $(N-L) \times 1$ vector $\mathbf{b}$. The direct estimation requires the storage of the complex $(N-L) \times(L+2)$ matrix $\mathbf{R}^{+}$

Consequently, our DC and channel estimation method is more efficient than (8). Moreover, we have separate explicit equations for the estimation of $\mathbf{h}$ and $\mathbf{d}$. In the next section we study the sensitivity of the joint DC and channel estimator with respect to noise and IQ gain/phase errors.

\section{ESTIMATION ERRORS}

In the previous section we described the maximum likelihood DC and channel estimator for a signal without IQ gain/phase error, i. e. $\eta=0$. In this section we analyze the error of this estimator for a signal with additive white Gaussian noise and with IQ gain/phase error, i. e. $\eta \neq 0$. In other words: while IQ imbalance is not addressed by the estimator, the error terms do consider IQ imbalance of the signal.

\section{A. DC Estimation Errors}

DC estimation errors have impact on the channel estimation. Moreover, for the equalization the DC-compensated samples $\mathbf{x}-\mathbf{a} \hat{d}$ are used. They are directly biased by an error of the DC estimation. Therefore, we first discuss the DC estimation errors.

From Equations (5) and (9) as well as from $\mathbf{P}_{\mathbf{T}}^{\perp} \mathbf{T}=\mathbf{0}$ we derive the DC estimation error

$$
\hat{d}-d=\underbrace{\eta \frac{\mathbf{a}^{H} \mathbf{P}_{\mathbf{T}}^{\perp} \mathbf{A}^{2} \mathbf{T h}^{*}}{\left\|\mathbf{P}_{\mathbf{T}}^{\perp} \mathbf{a}\right\|^{2}}}_{e_{\hat{d}, \eta}}+\underbrace{\frac{\mathbf{a}^{H} \mathbf{P}_{\mathbf{T}}^{\perp} \mathbf{n}}{\left\|\mathbf{P}_{\mathbf{T}}^{\perp} \mathbf{a}\right\|^{2}}}_{e_{\hat{d}, \mathbf{n}}} .
$$

First we look at the DC estimation error due to noise $e_{\hat{d}, \mathbf{n}}$. Using Equation (6) and because of $\mathrm{E}\left[\mathbf{w w}^{H}\right]=N_{0} \mathbf{I}$ and $\mathrm{E}\left[\mathbf{w w}^{T}\right]=0$ for complex Gaussian noise, we obtain after a few calculations

$$
\mathrm{E}\left[\mathbf{n n}^{H}\right]=\left(1+|\eta|^{2}\right) N_{0} \mathbf{I} .
$$

Consequently, the energy of this error term is given by

$$
E_{\hat{d}, \mathbf{n}}=\mathrm{E}\left[\left|e_{\hat{d}, \mathbf{n}}\right|^{2}\right]=\frac{N_{0}\left(1+|\eta|^{2}\right)}{\left\|\mathbf{P}_{\mathbf{T}}^{\perp} \mathbf{a}\right\|^{2}}
$$

and in case of no IQ-imbalance $(\eta=0)$ it is equivalent to the corresponding expression in [4]. The resulting noise suppression $S_{\hat{d}, n}=N_{0} / E_{\hat{d}, \mathbf{n}}$ of the DC estimator for different training sequence codes (TSCs) is listed in Table I, (a)+(b). Here and in the following, "noise suppression" values of an estimator are the ratio of the noise energy to the estimation error energy caused by noise (see also $S_{\hat{d}, n}$ in Fig. 2).

Next we have a look at the sensitivity to IQ gain/phase imbalance. The error energy is

$$
E_{\hat{d}, \eta}=|\eta|^{2} \frac{\left\|\mathbf{a}^{H} \mathbf{P}_{\mathbf{T}}^{\perp} \mathbf{A}^{2} \mathbf{T} \mathbf{h}^{*}\right\|^{2}}{\left\|\mathbf{P}_{\mathbf{T}}^{\perp} \mathbf{a}\right\|^{4}}
$$

and is a function of the impulse response $\mathbf{h}$. To quantify the error energy without assumptions on $\mathbf{h}$, we replace the error energy by the maximum error energy using the Schwarz inequality. The maximum error energy is

$$
E_{\hat{d}, \eta}^{\max }=\frac{|\eta|^{2}\|\mathbf{h}\|^{2}}{\left\|\mathbf{P}_{\mathbf{T}}^{\perp} \mathbf{a}\right\|^{4}}\left\|\mathbf{a}^{H} \mathbf{P}_{\mathbf{T}}^{\perp} \mathbf{A}^{2} \mathbf{T}\right\|^{2} \geq E_{\hat{d}, \eta}
$$

To characterize the sensitivity to IQ imbalance the minimum imbalance suppression

$$
S_{\hat{d}, \eta}^{\min }=|\eta|^{2}\|\mathbf{h}\|^{2} / E_{\hat{d}, \eta}^{\max }
$$

is used. Table I, (c)+(d), summarize the minimum suppression values. Similar to noise suppression, the "imbalance suppression" is defined as the ratio of the "imbalance error energy" 
TABLE I

SUPPRESSION PARAMETERS OF THE DC AND CHANNEL ESTIMATION

\begin{tabular}{|c|c|c|c|c|c|c|c|c|}
\hline tsc & 0 & 1 & 2 & 3 & 4 & 5 & 6 & 7 \\
\hline$L$ & \multicolumn{8}{|c|}{ (a) $S_{\hat{d}, n}[\mathrm{~dB}](\mathrm{GMSK})$} \\
\hline 4 & 12.5 & 12.3 & 12.5 & 12.3 & 13.3 & 12.4 & 13.3 & 12.9 \\
\hline 5 & 12.0 & 11.9 & 12.0 & 11.9 & 13.0 & 12.1 & 13.1 & 12.6 \\
\hline 6 & 11.7 & 11.5 & 11.7 & 11.5 & 12.6 & 11.9 & 12.8 & 12.2 \\
\hline$L$ & \multicolumn{8}{|c|}{ (b) $S_{\hat{d}, n}[\mathrm{~dB}](8 \mathrm{PSK})$} \\
\hline 4 & 11.9 & 11.9 & 9.5 & 9.5 & 9.4 & 9.4 & 9.5 & 9.1 \\
\hline 5 & 11.0 & 11.2 & 8.3 & 8.3 & 8.0 & 8.1 & 8.1 & 7.7 \\
\hline 6 & 9.8 & 10.0 & 6.6 & 6.7 & 6.2 & 6.2 & 6.2 & 6.0 \\
\hline$L$ & \multicolumn{8}{|c|}{ (c) $S_{\hat{d}, \eta}^{\min }[\mathrm{dB}](\mathrm{GMSK})$} \\
\hline 4 & 4.2 & 3.9 & 4.2 & 3.9 & 11.2 & 12.1 & 12.0 & 5.3 \\
\hline 5 & 2.7 & 2.6 & 2.7 & 2.6 & 10.6 & 12.3 & 10.9 & 4.4 \\
\hline 6 & 2.1 & 2.2 & 2.1 & 2.2 & 9.5 & 9.8 & 8.6 & 3.8 \\
\hline$L$ & \multicolumn{8}{|c|}{ (d) $S_{\hat{d}, \eta}^{\min }[\mathrm{dB}](8 \mathrm{PSK})$} \\
\hline 4 & 4.3 & 4.3 & -1.2 & -1.2 & -0.3 & -0.3 & -1.0 & -0.6 \\
\hline 5 & 1.1 & 1.0 & -1.5 & -1.8 & -2.9 & -2.4 & -2.8 & -2.0 \\
\hline 6 & -0.6 & -1.1 & -1.3 & -1.6 & -4.9 & -4.5 & -4.7 & -2.6 \\
\hline$L$ & \multicolumn{8}{|c|}{ (e) $S_{\hat{\mathbf{h}}, n}[\mathrm{~dB}](\mathrm{GMSK})$} \\
\hline 4 & 19.7 & 18.4 & 19.7 & 18.4 & 27.4 & 19.3 & 29.3 & 22.2 \\
\hline 5 & 18.0 & 17.6 & 18.0 & 17.6 & 25.6 & 18.9 & 27.4 & 21.2 \\
\hline 6 & 17.3 & 16.8 & 17.3 & 16.8 & 23.2 & 18.8 & 25.3 & 20.1 \\
\hline$L$ & \multicolumn{8}{|c|}{ (f) $S_{\hat{\mathbf{h}}, n}[\mathrm{~dB}](8 \mathrm{PSK})$} \\
\hline 4 & 16.4 & 16.4 & 12.4 & 12.4 & 11.5 & 11.8 & 11.6 & 11.1 \\
\hline 5 & 14.2 & 14.9 & 10.6 & 10.7 & 9.5 & 10.1 & 9.7 & 9.3 \\
\hline 6 & 12.0 & 12.4 & 8.2 & 8.5 & 7.2 & 7.6 & 7.3 & 7.4 \\
\hline$L$ & \multicolumn{8}{|c|}{ (g) $S_{\hat{\mathbf{h}}, \eta}^{\min }[\mathrm{dB}](\mathrm{GMSK})$} \\
\hline 4 & 11.4 & 10.1 & 11.4 & 10.1 & 25.3 & 19.1 & 28.0 & 14.7 \\
\hline 5 & 8.6 & 8.3 & 8.6 & 8.3 & 23.3 & 19.2 & 25.2 & 13.0 \\
\hline 6 & 7.7 & 7.5 & 7.7 & 7.5 & 20.1 & 16.8 & 21.1 & 11.6 \\
\hline$L$ & \multicolumn{8}{|c|}{ (h) $S_{\hat{\mathbf{h}}, \eta}^{\min }[\mathrm{dB}](8 \mathrm{PSK})$} \\
\hline 4 & 8.8 & 8.8 & 1.7 & 1.7 & 1.8 & 2.2 & 1.1 & 1.4 \\
\hline 5 & 4.3 & 4.7 & 0.8 & 0.6 & -1.3 & -0.3 & -1.2 & -0.4 \\
\hline 6 & 1.6 & 1.4 & 0.4 & 0.2 & -3.9 & -3.1 & -3.7 & -1.2 \\
\hline
\end{tabular}

$|\eta|^{2}\|\mathbf{h}\|^{2}$ to the estimation error energy caused by the IQ imbalance $E_{\hat{d}, \eta}$ (see also $S_{\hat{d}, \eta}$ in Fig. 2). We denote the energy of the error added to the received signal by IQ imbalance, i. e. the energy of the mirror signal, as "imbalance error energy".

We see that modulation types and training sequences with small noise suppression $S_{\hat{d}, n}$ also have a small IQ imbalance suppression $S_{\hat{d}, \eta}$. The same is true for large suppression values. However, the order is not exactly the same. Moreover, the values for the noise suppression $S_{\hat{d}, n}$ are always several dB larger than for the IQ imbalance suppression $S_{\hat{d}, \eta}$. Consequently, if the DC offset $d$ and the energy of the mirror signal $\eta \mathbf{T h}^{*}$ are of the same order of magnitude, the DC estimation error due to IQ imbalance is dominant.

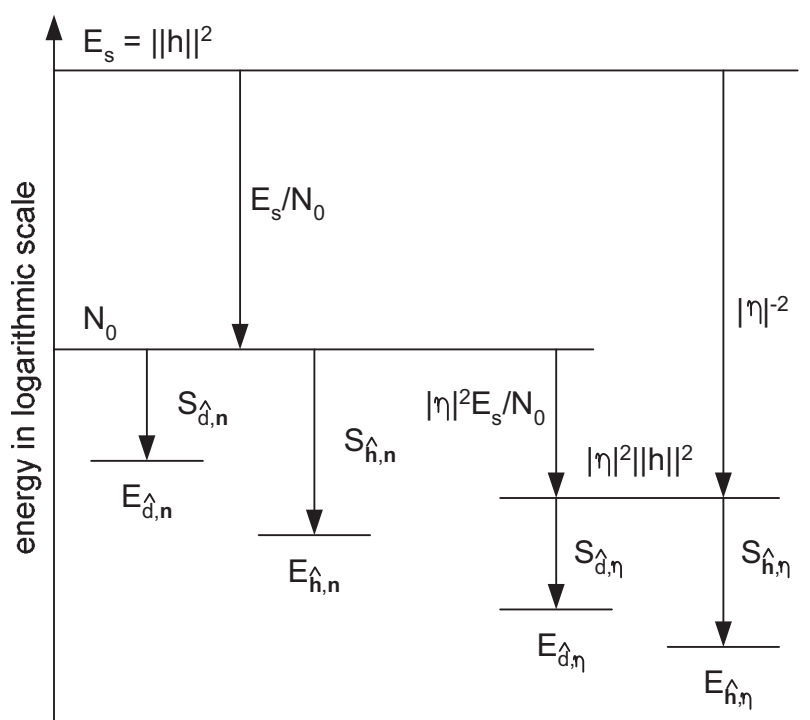

Fig. 2. Level diagram to explain the contribution of the different error and suppression terms.

\section{B. Channel Estimation Errors}

The channel estimation is biased by the DC estimation error according to Equation (10). This bias can be split into two error terms. The error due to noise is given by $\mathbf{e}_{\hat{\mathbf{h}}, \mathbf{n}}=\mathbf{T}^{+} \mathbf{a} e_{\hat{d}, \mathbf{n}}$ and the error due to IQ imbalance is given by $\mathbf{e}_{\hat{\mathbf{h}}, \eta}=\mathbf{T}^{+} \mathbf{a} e_{\hat{d}, \eta}$. The noise suppression $S_{\hat{\mathbf{h}}, n}$ and the minimum imbalance suppression $S_{\hat{\mathbf{h}}, \eta}^{\min }$ of the channel estimator are calculated in the same way as for the DC estimator:

$$
\begin{aligned}
S_{\hat{\mathbf{h}}, n} & =N_{0}\left(1+|\eta|^{2}\right) / E_{\hat{\mathbf{h}}, \mathbf{n}}, \\
S_{\hat{\mathbf{h}}, \eta} \min & =|\eta|^{2}\|\mathbf{h}\|^{2} / E_{\hat{\mathbf{h}}, \eta}^{\max } .
\end{aligned}
$$

The results are presented in Table I, (e)-(h).

We can see that the noise suppression is excellent for GMSK (more than $17 \mathrm{~dB}$ ). However, for 8PSK the noise suppression for training sequence numbers $2-7$ is roughly $4 \mathrm{~dB}$ weaker than for training sequence numbers 0 and 1 . For GMSK the IQ imbalance suppression is always more than $7.5 \mathrm{~dB}$ so that the error due to IQ imbalance is negligible.

Besides these two error terms, IQ imbalance and noise add errors to the channel estimators even in case of an error-free DC estimator. Fig. 2 shows all error and suppression terms in a level diagram. With the values in Table I and values of $E_{s} / N_{0}$ as well as $\eta$, the different contributions can be compared for any zero IF receiver using joint channel and DC estimation.

For example, a receiver with an input signal level of $E_{s}=$ $-90 \mathrm{dBm}$, a noise level $N_{0}=-110 \mathrm{dBm}$ (equivalent noise bandwidth $270 \mathrm{kHz}$ ) and $\eta=0.025(1+j$ ) (corresponding to $0.44 \mathrm{~dB}$ gain imbalance and $2.9^{\circ}$ phase error), i. e. $|\eta|^{2} E_{s}=$ $-119 \mathrm{dBm}$, has the following additional error contributions for TSC 0 and TSC 7, respectively.

$$
\begin{array}{rll}
E_{\hat{d}, n} / \mathrm{dBm} & =-121.9, & -119.1 \\
E_{\hat{\mathbf{h}}, n}^{\max } / \mathrm{dBm} & =-126.4, & -121.1 \\
E_{\hat{d}, \eta} / \mathrm{dBm} & =-123.3, & -119.6 \\
E_{\hat{\mathbf{h}}, \eta}^{\max } / \mathrm{dBm} & =-127.8, & -120.4
\end{array}
$$



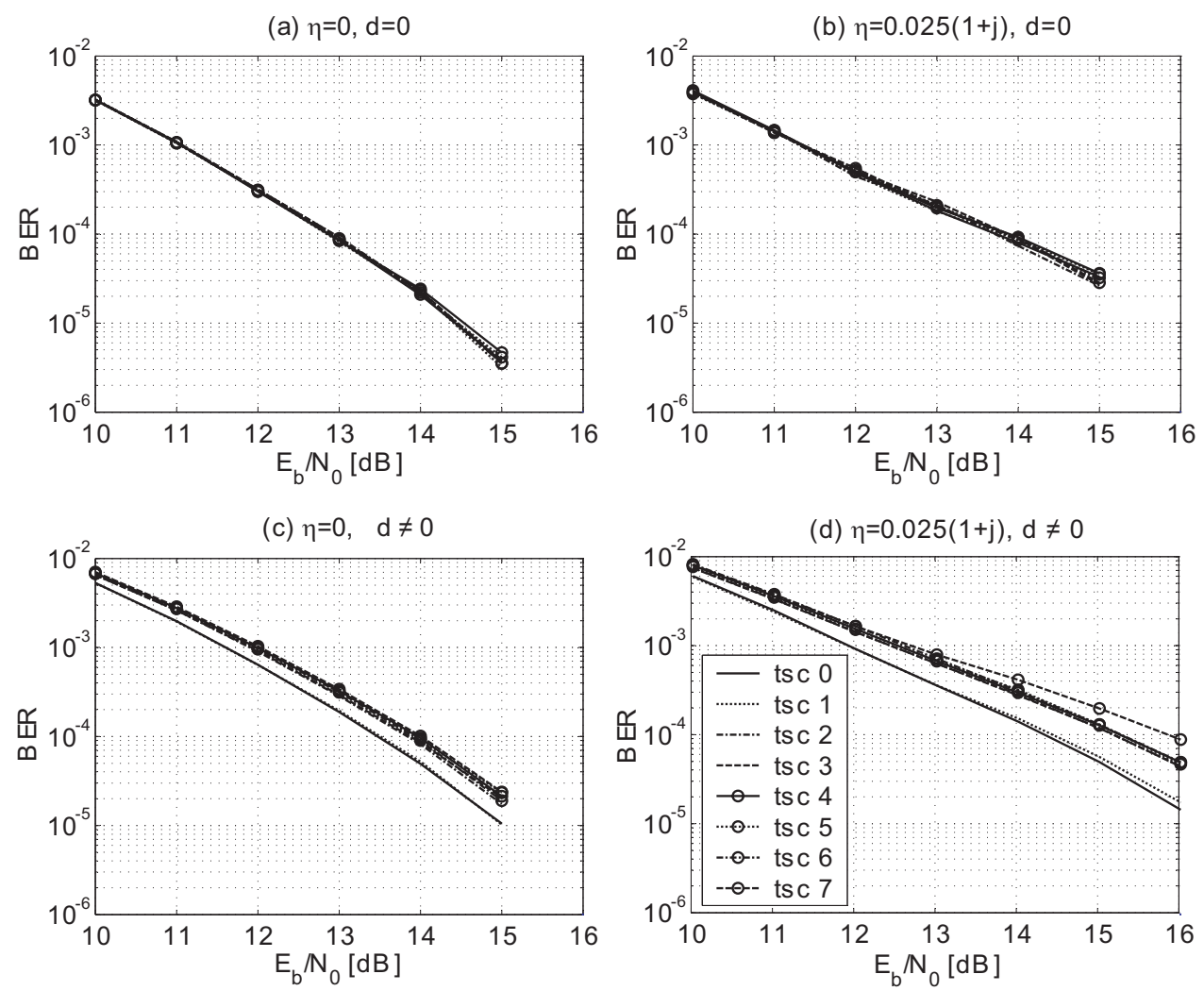

Fig. 3. Bit error rate as a function of $E_{b} / N_{0}$ for training sequences 0 to 7 .

The numbers suggest that the loss for training sequence code number 7 should be more than $0.5 \mathrm{~dB}$ higher than for training sequence code number 0 . In the next subsection, bit error rate simulations confirm this suggestion.

\section{Bit Error Rates}

To illustrate the effects of the derived DC and channel estimation errors, bit error rate simulations were performed. For each simulation 100,000 GSM/EDGE compliant 8PSK bursts with random data bits were generated. Noise, DC offset, and IQ imbalance were added and different training sequences were used. The receiver is very similar to the one presented in [1], except that no noise whitening prefilter was used. The simulated receiver is fully compliant with the GSM/EDGE standard for all training sequence codes. A static channel was chosen to have a constant channel impulse response. Note, that although the physical channel impulse response is a Dirac pulse the overall channel impulse response $h_{k}$ is spread over several taps due to pulse shaping and filtering. In GSM/EDGE the pulse shape is not designed for Nyquist filtering.

Fig. 3 shows the resulting bit error rate curves. The ratio of signal energy per bit $E_{b}$ and the white noise Energy $N_{0}$ were used as the abscissa. Note that for 8PSK the energy per bit is $4.8 \mathrm{~dB}$ below the signal energy per symbol. In the first simulation, the DC offset and IQ imbalance were set to zero. Also the receiver assumes a zero DC offset. The resulting bit error rate curves are shown in Fig. 3(a). Introducing an IQ imbalance with $\eta=0.025(1+j)$ corresponding to $0.44 \mathrm{~dB}$ gain imbalance and $2.9^{\circ}$ phase error leads to Fig. 3(b). In
Fig. 3(c), the receiver estimates and compensates the non-zero DC offset but the IQ imbalance is zero. In Fig. 3(d) both, IQ imbalance and DC offset are present.

To compare the different bit error rate curves, those values for $E_{b} / N_{0}$ that are required to have a bit error rate of $10^{-3}$ and $10^{-4}$, respectively, were calculated by log-linear interpolation. The values are summarized in Table II. Without DC and IQ imbalance, the bit error performance is almost identical for all training sequence codes. The same holds for the case of no DC but with IQ imbalance although a loss of up to $1 \mathrm{~dB}$ can be seen for a bit error rate as low as $10^{-4}$. If the IQ imbalance is zero but DC estimation is introduced, a loss of up to $1.1 \mathrm{~dB}$ arises.

As was expected from the estimated error energy and suppression values, the loss is different for different training sequences. The mutual difference has a maximum value of $0.5 \mathrm{~dB}$. Adding IQ imbalance leads to a maximum difference of $1.5 \mathrm{~dB}$. The loss in sensitivity for a bit error rate as low as $10^{-4}$ varies from $1.5 \mathrm{~dB}$ to $3.0 \mathrm{~dB}$.

In the last two cases, training sequence numbers 0 and 1 are significantly better than numbers 2 to 7 , whereas number 7 is the worst. The bit error results match well with the suppression values derived in the previous two subsections.

\section{CONCLUSion}

We analyzed a maximum likelihood joint DC and channel estimator for GSM/EDGE direct conversion receivers. We derived estimation errors caused by noise and IQ gain/phase 
TABLE II

REQUIRED $E_{b} / N_{0}$ TO HAVE A BIT ERROR RATE OF $10^{-3}$ AND $10^{-4}$ WITH THE SIMULATED 8PSK RECEIVER, RESPECTIVELY

\begin{tabular}{r||r|r|r|r|r|r|r|r}
\hline \multicolumn{1}{r||}{ tsc } & 0 & 1 & 2 & 3 & 4 & 5 & 6 & 7 \\
\hline \hline BER & (a) $\eta=0, \quad \hat{d}=d=0$ \\
\hline $10^{-3}$ & 11.1 & 11.1 & 11.1 & 11.1 & 11.1 & 11.0 & 11.0 & 11.0 \\
$10^{-4}$ & 12.9 & 12.9 & 12.9 & 12.9 & 12.9 & 12.9 & 12.9 & 12.9 \\
\hline BER & (b) $\eta=0.025(1+j), \quad d \neq 0$ \\
\hline $10^{-3}$ & 11.3 & 11.3 & 11.3 & 11.3 & 11.4 & 11.3 & 11.4 & 11.3 \\
$10^{-4}$ & 13.7 & 13.8 & 13.8 & 13.8 & 13.9 & 13.9 & 13.8 & 13.9 \\
\hline BER & (c) $\eta=0$, & $d \neq 0$ \\
\hline $10^{-3}$ & 11.6 & 11.6 & 11.9 & 11.9 & 12.0 & 12.0 & 12.0 & 12.0 \\
$10^{-4}$ & 13.5 & 13.5 & 13.8 & 13.9 & 14.0 & 13.9 & 13.9 & 14.0 \\
\hline BER & (d) $\eta=0.025(1+j), d \neq 0$ \\
\hline $10^{-3}$ & 12.0 & 12.0 & 12.5 & 12.5 & 12.6 & 12.6 & 12.6 & 12.8 \\
$10^{-4}$ & 14.4 & 14.5 & 15.2 & 15.2 & 15.3 & 15.3 & 15.3 & 15.9 \\
\hline
\end{tabular}

imbalance. It turns out that the selection of the training sequence has a significant influence on these errors.

The influence of those error terms on the bit error rate of the receiver was demonstrated by Monte-Carlo receiver simulations. Differences of up to $1.5 \mathrm{~dB}$ for a targeted bit error rate of $10^{-4}$ in a static (but non-zero order) channel were shown. Bit error rate results can only be determined for specific RF transceivers and specific equalizer algorithms. Some equalizers can be more sensitive to DC estimation errors due to noise while others may be more sensitive to channel estimation errors due to IQ imbalance. Nevertheless, we can derive the following rough conclusions:

A symbol-by-symbol rotation by $\phi=3 \pi / 8$ leads to significant DC estimation, channel estimation, and bit errors for most training sequence codes. Consequently, a rotation by $\phi=\pi / 2$ for both, GMSK and 8PSK would allow a better 8PSK receiver performance. However, in EDGE the receiver has to detect the modulation without additional signalling. This would be more difficult, if the rotation angle is the same for GMSK and 8PSK. Nevertheless, choices like $\phi=\pi / 4$ would decrease the estimation errors as can be easily shown using the derived expressions.

For 8PSK training sequence numbers 0 and 1 lead to better detection results than other training sequences. This means that bit/block error rate simulations and measurements with bursts using one training sequence do not accurately represent simulations and measurements with bursts using other sequences. EDGE receiver simulation results without information about the training sequence have to be questioned critically.

\section{REFERENCES}

[1] W. H. Gerstacker and R. Schober, "Equalization concepts for EDGE," IEEE Trans. Wireless Commun., vol. 1, no. 1, pp. 190-199, Jan. 2002.

[2] B. Lindoff, "Using a direct conversion receiver in EDGE terminals: a new DC offset compensation algorithm," in Proc. PIMRC 2000, pp. 959963, Sept. 2000.

[3] B. Lindoff and P. Malm, "BER performance analysis of a direct conversion receiver," IEEE Trans. Commun., vol. 50, no. 5, pp. 856-865, May 2002.

[4] D. Hui, B. Lindoff, and K. Zangi, "Enhanced DC estimation via sequence-specific frequency offset," in 2002 IEEE 56th Vehicular Technology Conference Proceedings. VTC 2002, pp. 161-165, Sept. 2002

[5] 3rd Generation Partnership Project (3GPP), TSG GSM/EDGE Radio Access Network, 45 series (Radio aspects), 2003.

[6] J. K. Cavers and M. W. Liao, "Adaptive compensation for imbalance and offset losses in direct conversion transceivers," IEEE Trans. Veh. Technol., vol. 42, no. 4, pp. 581-587, Nov. 1993.

[7] G. T. Gil, I. H. Sohn, J. K. Park, and Y. H. Lee, "Joint ML estimation of carrier frequency, channel, I/Q mismatch, and DC offset in communication receivers," IEEE Trans. Veh. Technol., vol. 54, no. 1, pp. 338-349, Jan. 2005. 